\title{
TOWARDS A REFERENCE MODEL FOR COLLABORATIVE NETWORKED ORGANIZATIONS
}

\author{
Luis M. Camarinha-Matos ${ }^{1}$, Hamideh Afsarmanesh ${ }^{2}$ \\ ${ }^{1}$ New University of Lisbon, PORTUGAL, cam@uninova.pt \\ ${ }^{2}$ University of Amsterdam, NETHERLANDS, hamideh@science.uva.nl
}

\begin{abstract}
A growing number of collaborative networked organizations can be found in industry, services, and research. However, the lack of a reference model that could synthesize and formalize the base concepts, principles, and recommended practices, is an obstacle for an easier and more consistent development of the area. Therefore a reference modeling approach is proposed considering multiple modeling perspectives. Examples are given and steps for firther research are identified. Establishing a reference model in this area is a long term endeavor; this contribution is aimed as just a step in this process.
\end{abstract}

\section{INTRODUCTION}

Collaborative networks are emerging in a large variety of forms, including virtual organizations, virtual enterprises, dynamic supply chains, professional virtual communities, virtual organization breeding environments, collaborative virtual laboratories, etc. [1], [2], [3]. Although not all, most forms of collaborative networks imply some kind of organization over the activities of their constituents, identifying roles for the participants, and some governance rules. Therefore, these can be called manifestations of collaborative networked organizations (CNOs). A large body of empiric knowledge related to collaborative networked organizations is already available, but there is an urgent need to consolidate this knowledge and build the foundations for a more sustainable development of this area.

Lack of reference models for collaborative networked organizations or even to some of their manifestations (such as virtual enterprises) is a common concern found in the literature, being also pointed out as an obstacle for a more consistent development of the area [4]. The difficulties are found namely in the terminology and associated meanings, what leads to frequent misunderstandings among members of this research community with a different original background.

Establishing a reference model for a new entity is not an easy task when only limited background inputs are available. In this context the reference model shall play a guiding / visionary role. Once established, the reference model defines a common basis for understanding and explaining (at least at a high level of abstraction) the different manifestations of the paradigm. It shall facilitate the 
development of particular models for specific CNOs (Figure 1). These particular models will drive the implementations and serve also to simulate / evaluate concrete networks.

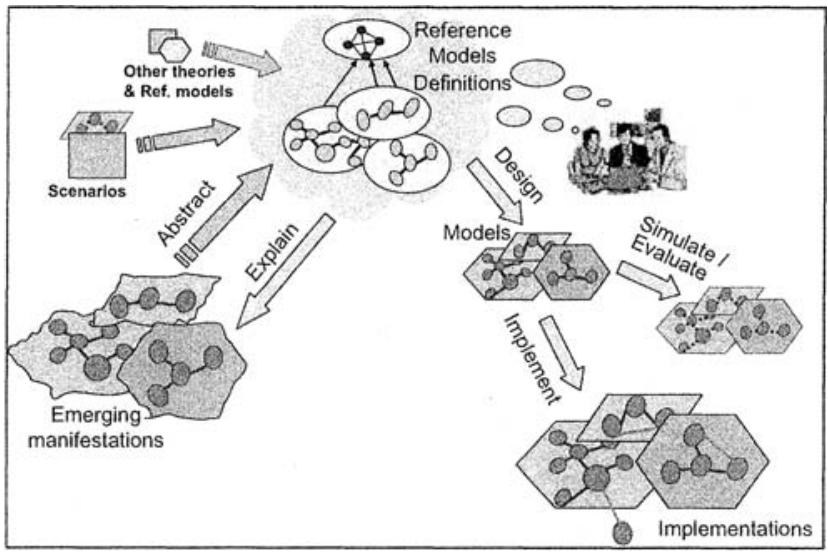

Figure 1 - Reference models in a context

\section{EARLY CONTRIBUTIONS}

When attempting to establish a reference model it is fundamental to consider the potential inputs and partial contributions from previous works. In fact some previous and ongoing projects have tried to contribute to reference models of some manifestations of collaborative networks, namely for Virtual enterprises / virtual organizations (VE/VO).

Figure 2 illustrates the diversity of sources which can potentially be used as inputs to this activity. As shown, there are two main streams:

- Enterprise-centric stream, which starts from the extensive past modeling activities at enterprise level and try to incrementally extend / adapt such models to the context of networks of enterprises.

- Network-centric stream, which puts the emphasis primarily on the networks and their properties, rather than on the characteristics of the individual elements.

These streams are not totally disjunctive and several initiatives show in fact partial elements of the two perspectives.

The approaches to modeling very much depend on the dominant background of people involved in each initiative. Three main groups or "schools" encompass most of the past VE/VO related developments:

i) Enterprise modeling, based on the underlying "culture" represented by the Zachman framework, GRAI-GIM, PERA, CIM-OSA, GERAM, and related developments [10].

ii) Organizational / management school, which departs from traditional organizational structures such as supply chains and the corresponding SCOR model, and tries to reason about emerging organizational patterns in new collaborative forms. 
iii) VE/VO ICT-based projects, which put a strong emphasis on the ICT tools and infrastructures to support collaboration. A large number of projects have been carried out in this area that, although showing a "fragmented" and mostly ad-hoc approach, contribute with partial elements to better understand CNOs, their modeling needs and possible approaches.

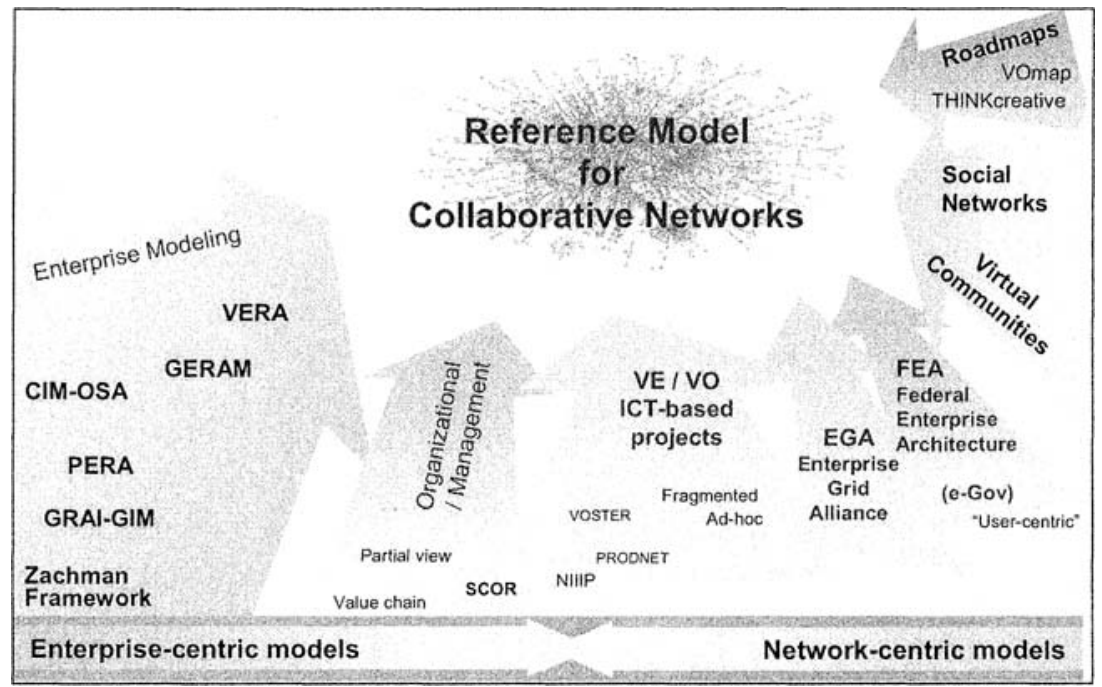

Figure 2 - Main inputs to the design of a CNO reference model

Some survey works analyzing early contributions namely in the areas i) and iii) above can be found in the literature, such as [11], [12]. The PRODNET project [4] or the VITE model [6] are examples of ICT-driven initiatives. An example survey under perspective ii) was conducted in the VOSTER project [9], which also included some analysis of ICT developments and common practices on VE/VO implementation [4]. Other areas of interest include:

iv) Grid community, which has been moving towards virtual organizations and is now trying to consider a business perspective, as in the case of the Enterprise Grid Architecture initiative [7].

v) E-Government, which represents a wide area but that has some common elements when it addresses the cooperation among different governmental organizations, as illustrated by the Federal Enterprise Architecture [8].

vi) Social networks and virtual communities are areas that although not yet offering much in terms of reference models, have developed considerable background in terms of the basic properties of networks with a strong basis on graph theory.

vii) Collaborative networks roadmapping initiatives such as THINKcreative, VOmap and others which have contributed to the identification of the research challenges in the area [2].

Figure 3 tries to put into a simplified historic perspective some of the key initiatives and events that represent a substantial input to a better understanding of collaborative networks and therefore offer base material for the elaboration of reference models for CNOs. 


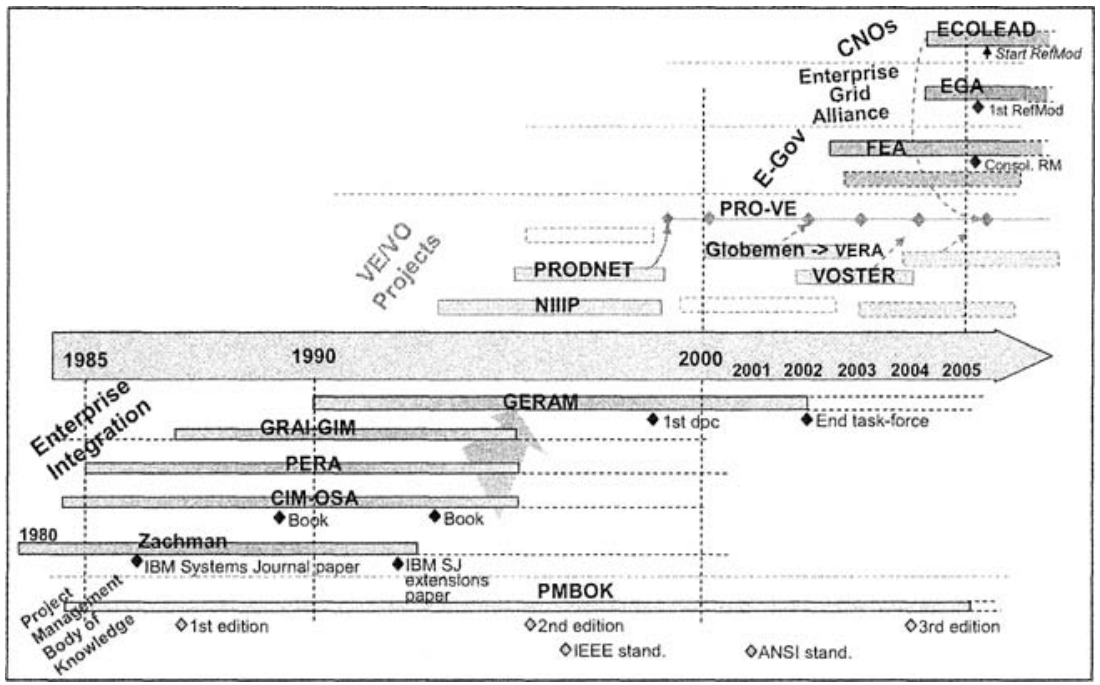

Figure 3 - Towards a CNO reference model - A simplified historic perspective

The lower half of the diagram in Figure 3 includes major representatives of the enterprise integration and modeling area that were particularly active in the 80 s and 90s. A parallel initiative, from a different area but that can also give some hints for some cases of virtual organizations, is the Project Management Body of Knowledge.

The upper half of the diagram shows initiatives that are more directly related to collaborative networks. Of particular relevance here is the heritage of a large number of VE/VO projects. VOSTER represented an attempt to synthesize part of this heritage. The PRO-VE series of conferences and the corresponding proceedings are also playing a major role in the consolidation of knowledge in the area and contributing to establish some (progressive) consensus, important elements towards the definition of reference models.

\section{BASE TERMINOLOGY}

The establishment of reference models for CNOs is frequently pointed out as a major need for the consolidation and sustainable development of the area. However it seems that there is not much consensus on what this term exactly means. In fact it seems that it represents quite different things for different people and consequently it raises quite different expectations regarding its utility. It is therefore necessary to revisit the concept of reference model and its purpose.

The clarification of the base concepts is however not that easy as the literature in this area is full of confusing terminology. To refer only a few, it is common to find terms such as reference architecture, reference framework, architectural framework, system architecture, etc. often used with similar or largely overlapping meanings. Without the aim of giving a "final" definition, the following working definitions are suggested:

- Model: A model is an abstract representation of an environment, system, or 
entity in the physical, social, or logical world.

Typically a model refers only to some aspects of the phenomenon being modeled, and two models of the same phenomenon may be essentially different. This may be due to: different requirements, differences in conceptual approaches, esthetic preferences, and also different past experiences. Therefore, users of a model need to understand the model's purpose and the assumptions or limits of its validity. There can be models at various levels of abstraction, from very abstract theoretical constructs, to (detailed) representations very close to the modeled entity or implementation.

- Framework: In general a framework is a structure for supporting or enclosing something else. In the modeling area, a framework can be seen as an "envelope" that might include a number of (partial) models, collections of templates, procedures and methods, rules, and even tools (e.g. modeling languages).

- Reference model: A reference model is a generic abstract representation for understanding the entities and the significant relationships among those entities of some area, and for the derivation of other specific models for particular cases in that area. Preferably a reference model is based on a small number of unifying concepts and may be used for education, explaining, and systems' development.

A CNO reference model is thus a generic conceptual model that synthesizes and formalizes the base concepts, principles and recommended practices for collaborative networked organizations. It is intended as an authoritative basis (guide) to streamline or facilitate the creation of focused models for the various manifestations of CNOs as well as architectures and implementation models for particular systems development. A reference model is generic and not directly applicable to concrete cases but rather provides the basis for the development (derivation) of other models closer to those cases.

- Architecture: An architecture is an abstract description of a specific system, i.e. a particular model that even at a logical level tends to indicate the system structure, functions of its components, their interactions, and constraints, and can be used to develop the system. Architecture is focused on "building a system" and must be complete at its level of abstraction; therefore not all models are architectures. Although there is a difference between engineering and architecture (compare with roles of civil engineer and building architect), to some extent the architecture depends on engineering principles and available technology. An architecture can be formulated in a descriptive or in a prescriptive style. Descriptive style defines an enumeration of design elements and formal "arrangements" between them. Prescriptive style establishes constraints, namely by limiting the possible design elements and their "arrangements".

- Reference architecture: A reference architecture aims at structuring the design of architectures for a given domain by defining a unified terminology, describing the functionality and roles of components, providing template components, giving example architectures, and defining a development methodology. It corresponds to architecture as a style or method in the sense that may represent a coherent set of design principles to be used in a specific area. The reference architecture is the basis for designing the specific architectures for particular instances of systems in the class of systems covered by the reference architecture. In the CNO domain, a reference architecture for VO management systems would represent the "structure" and principles to be followed by particular architectures of concrete 
VO management systems. The concept of reference architecture also induces the creation of generic re-usable "building blocks".

Based on the definition given above, two main "anchors" can be associated to a reference model: Authority and re-use (Figure 4).

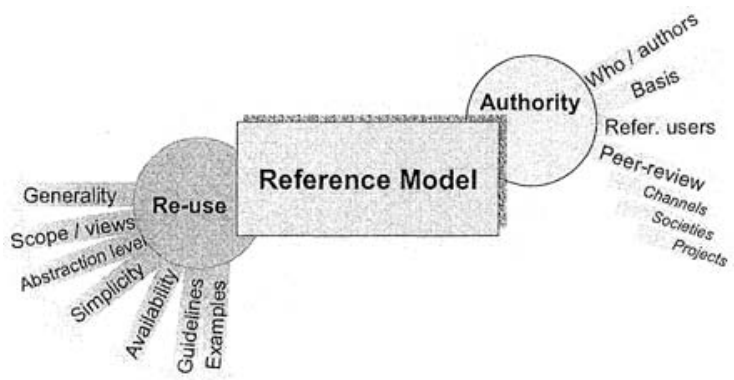

Figure 4 - Key anchors in a reference model

Establishing a model as an authoritative reference depends on a number of factors, including the authorship, i.e. the reputation / prestige of the involved contributors, the adopted bases and referenced sources, the list of early adopters or reference users, the quality of the peer reviewing process, and also the dissemination channels, professional societies and projects involved in its dissemination. Re-usability of the elements of a reference model, with the objective of streamlining the design and development of particular models, also depends on a number of factors, including: the generality of the model, its scope and covered views, the abstraction level and simplicity, the forms of availability / easiness of access to supporting information, the existence of guidelines for use and examples of application to typical cases.

It is also important to distinguish between reference models and standards. Both share some common aspects (Figure 5), namely aiming at simplifying the creation of new systems and providing some stable conceptual background or building blocks. Regarding the process, both start with building consensus but then they evolve into different directions.

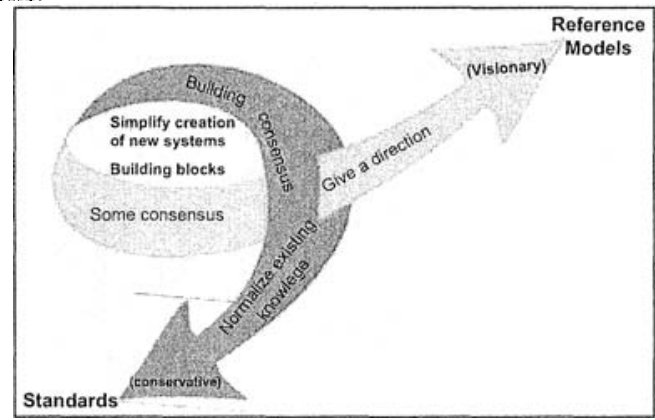

Figure 5 - Reference models and standards 


\section{REFERENCE MODELING APPROACH}

A complex entity such as a CNO can be observed and analyzed through different lenses or perspectives. Each lens can provide complementary elements that help in achieving a better understanding of the paradigm. It is however important to note that lenses might also cause distortions. Particularly if one tries to explain all aspects of CNOs through the perspective of a single lens, not only it leads to dangerous over-simplifications, but even introduces some misconceptions. Therefore a holistic perspective is needed.

Most of the previous publications towards a reference model for a $\mathrm{CNO}$ (or some of its manifestations) are either technology-biased (e.g. [11]), or business-biased (e.g. [9]). A holistic approach, combining both perspectives would guarantee a better alignment of business and technology. On the other hand, we shall not ignore other aspects such as culture, values, norms and principles, trust, etc. that can represent another dimension - the "style" of the CNO (a term borrowed from the area of architecture / civil construction). These aspects are less addressed in previous modeling works but shall be considered in a holistic reference model for CNOs.

When modeling a $\mathrm{CNO}$, it is important to consider both its internal and external aspects (Fig. 6).

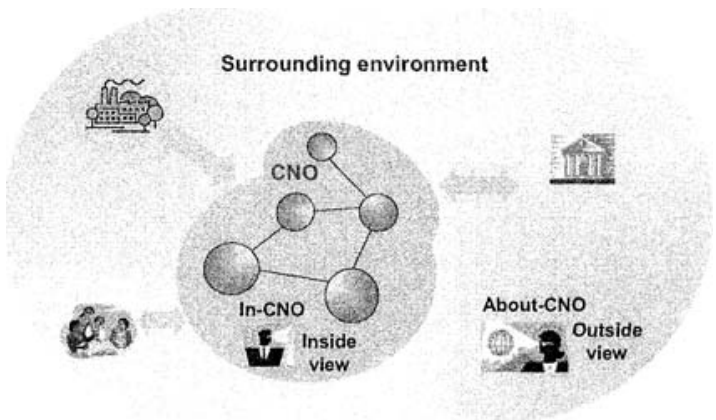

Figure 6-Modeling perspectives

Therefore in ARCON (A Reference Model for COllaborative Networks), being developed within the ECOLEAD project [5], [1], these two subspaces comprehensively cover the internal (In-CNO) aspects of CNOs as well as the external (About-CNO) aspects that are related to the logical surrounding of CNOs.

In-CNO perspective. This perspective aims at providing of an abstract representation of the $\mathrm{CNO}$ from inside, namely the identification of a set of characteristic properties that can together capture the elements constituting CNOs. Building In-CNO abstract representation is challenging due to the large number of distinct and varied entities, roles, concepts, functionality, rules and regulations, etc. inside the CNOs. To better characterize these aspects, four dimensions are proposed and defined to cover the In-CON perspective, as follows:

- Structural dimension. This perspective addresses the structure or composition of the $\mathrm{CNO}$ in terms of its constituting elements (participants and their relationships) as well as the roles performed by those elements and other 
characteristics of the network nodes such as the location, time, etc. This perspective is used in many disciplines (e.g. systems engineering, software engineering, economy, politics, cognitive sciences, manufacturing), although with different "wording" and tools.

- Componential dimension. This dimension focuses on the individual tangible/intangible elements in the CNO's network, e.g. the resource composition such as human elements, software and hardware resources, information and knowledge. Not all these elements are "physical" in a strict sense but rather represent the "things" of which the network is built of. Furthermore, elemental dimension also consists of ontology and the description of the information/knowledge.

- Functional dimension. This perspective addresses the "base operations" available at the network and the execution of time-sequenced flows of operations (processes and procedures) related to the "operational phase" of the CNO life cycle.

- Behavioral dimension. This dimension addresses the principles, policies, and governance rules that drive or constrain the behavior of the $\mathrm{CNO}$ and its members over time. Included here are elements such as principles of collaboration and rules of conduct, contracts, conflict resolution policies, etc.

About-CNO perspective. This perspective aims at reaching an abstract representation of the $\mathrm{CNO}$ as seen from the outside, i.e. which characteristic properties the CNO reveals in its interaction with its "logical" surrounding environment. A CNO as a whole might interact with, influence, and be influenced by a number of "interlocutors", e.g. customers, competitors, external institutions, potential new partners. The interactions between the CNO and these external entities are quite different, the same as the way each of these entity groups looks at the CNO. In order to better characterize these differences, the following modeling dimensions are proposed for the external or About-CNO perspective:

- Market dimension. This dimension covers both the issues related to the interactions with "customers" (or potential beneficiaries) and "competitors". The customers' facet involves elements such as the transactions and established commitments (contracts), marketing and branding, etc. On the competitors' side issues such as market positioning, market strategy, policies, etc. can be considered. The purpose / mission of the $\mathrm{CNO}$, its value proposition, joint identity, etc. are also part of this dimension.

- Support dimension. Under this dimension the issues related to support services provided by third party institutions are to be considered. Examples include certification services, insurance services, training, external coaching, etc.

- Societal dimension. This dimension captures the issues related to the interactions between the $\mathrm{CNO}$ and the society in general. Although this perspective can have a very broad scope, the idea is to model the impacts the CNO has or potentially can have on the society (e.g. impact on employment, economic sustainability of a given region, potential for attraction of new investments) as well as the constraints and facilitating elements (e.g. legal issues, public body decisions, education level) the society provides to the CNO development.

- Constituency dimension. This perspective focuses on the interaction with the 
universe of potential new members of the $\mathrm{CNO}$, i.e. the interactions with those organizations that are not part of the CNO but that the CNO might be interested in attracting. Therefore, general issues like sustainability of the network, attraction factors, what builds / provides a sense of community, or specific aspects such as rules of adhesion and specific "marketing" policies for members, are considered here.

In addition to these perspectives, a CNO model can be defined at multiple levels of abstraction. Currently three levels are considered in ARCON:

- General concepts level - that includes the most general concepts and related relationships, common to all CNOs independently of the application domain.

- Specific modeling level - an intermediate level that includes more detailed models focused on different classes of CNOs.

- Implementation modeling level - that represents models of concrete CNOs.

Fig. 7 combines the addressed perspectives into a single diagram.

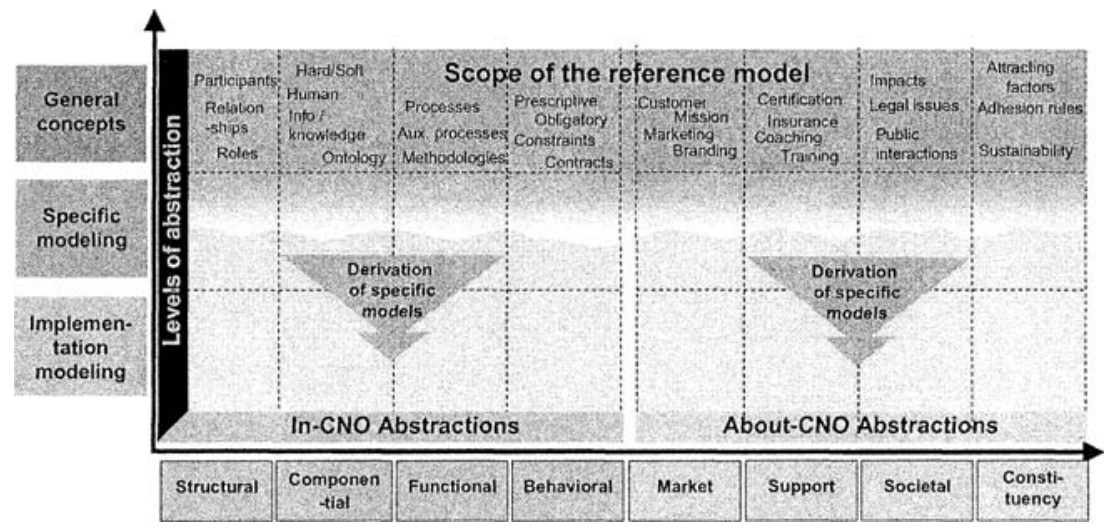

Figure 7 - A modelling framework for CNOs

Given the definition of reference model presented above, the scope of a CNO reference model covers mainly the "General Concepts" level and it might represent some elements of the "Specific Modeling" level. In other words, at the current stage of development of the CNO area, the first priority for a reference model is to consolidate the most general concepts that are common to all types of CNOs. With the progress in the area it will make sense to progressively consolidate more specific models for each major class of CNOs.

In terms of representation, a generic tool like UML, or another standard representation formalism (perhaps an ontology representation languages) that is proper for human understanding can be adequate for representation of the General Concepts level. For the other levels it will be necessary to consider other modeling tools and theories (e.g. set theory, graph theory, Petri nets, deontic logic, complexity theories, multi-agent systems, federated systems, etc.).

Another fundamental perspective to consider in the framework of $\mathrm{CNO}$ reference modeling is its life cycle. The following main stages can be considered: Creation, Operation, Evolution, and Metamorphosis or Dissolution. This is the subject of our ongoing work on development of ARCON, and will be addressed in future papers. 


\section{CONCLUSIONS AND FUTURE WORK}

The elaboration of a comprehensive reference model for CNOs is a very important element in the consolidation of existing knowledge in this area, and a basis for its consistent further progress. A number of early attempts have been made in various research projects, but frequently biased by a single perspective.

ECOLEAD is attempting to contribute to a more holistic reference model for CNOs considering multiple perspectives. Nevertheless it is clear that the establishment of a reference model capturing the variety of CNOs and their complexity is a long term endeavor that needs to start with a careful analysis of the current baseline and definition of related reference modeling frameworks. This paper presented our preliminary results in this direction as developed in the framework of an ongoing effort to establish A Reference Model for COllaborative Networks (ARCON).

\section{ACKNOWLEDGEMENTS}

This work was funded in part by the European Commission through the ECOLEAD project. The authors thank the contributions of their partners in this project.

\section{REFERENCES}

1. Afsarmanesh, H.; Camarinha-Matos, L.M. - A Framework for Management of Victual Organization Breeding Environments, in Proceedings of PRO-VE'05 - Collaborative Networks and their Breeding Environments, Springer, Valencia, Spain, 26-28 Sept 2005.

2. Camarinha-Matos, L.M.; Afsarmanesh, H. (Editors) - Collaborative Networked Organizations - A research agenda for emerging business models, Springer, 2004.

3. Camarinha-Matos, L.M.; Afsarmanesh, H. - Collaborative networks: A new scientific discipline, J. Intelligent Manufacturing, vol. 16, $\mathrm{N}^{\circ} 4-5, \mathrm{pp} 439-452,2005$.

4. Camarinha-Matos, L.M.; Afsarmanesh, H.; Ollus, M. (Editors) - Virtual Organizations - Systems and Practices, Springer, 2005

5. Camarinha-Matos, L.M.; Afsarmanesh, H.; Ollus, M. - ECOLEAD: A holistic approach to creation and management of dynamic virtual organizations, in Proceedings of PRO-VE'05 - Collaborative Networks and their Breeding Environments, Springer, pp. 3-16, Valencia, Spain, 26-28 Sept 2005.

6. Chalmeta, R. - Virtual transport enterprise integration, Transactions of the SDPS, Vol.4, N.4, Dec 2000. hip:/Www.sdpsnet.org/fournals/vol4-4/Ricardo-4.pdt

7. $\mathrm{EGA}$ - Enterprise Grid Alliance Reference Model, 13 Apr 2005. http/www gridalliance orgen workgroups/ReferenceModelasp

8. FEA - FEA Consolidated Reference Model, May 2005, http//Www whitehouse govonb/egovdocuntents/CRM.PDE

9. Katzy, B,; Zhang, C.; Loeh, H. - Reference models for virtual organisations, in Virtual organizations Systems and Practices, L. M. Camarinha-Matos, H. Afsarmanesh, M. Ollus (Editors), Springer, 2005.

10. Noran, O. - A mapping of individual architecture frameworks (GRAI, PERA, C4ISR, CIMOSA, ZACHMAN, ARIS) onto GERAM, in Handbook on enterprise architecture, P. Bernus, L. Nemes, G Schmidt (Ed.s), Springer, 2003.

11. Tolle, M.; Bernus, P. - Reference models supporting enterprise networks and virtual enterprises, International Journal of Networking and Virtual Organisations $2003-$ Vol. 2, No.1 pp. 2 - 15 . http/www inderscience.com/storage/f161185104291237.pdf

12. Tolle, M.; Bernus, P.; Vesterager, J. - Reference models for virtual enterprises, in Collaborative business ecosystems and virtual enterprises, L. M. Camarinha-Matos, Editor, Kluwer Academic Publishers, 2002. 